\title{
Near-Infrared Grating Couplers for Silicon Nitride Photonic Wires
}

\author{
Ananth Z. Subramanian, Shankar Selvaraja, Member, IEEE, Peter Verheyen, Ashim Dhakal, \\ Katarzyna Komorowska, and Roel Baets, Fellow, IEEE
}

\begin{abstract}
Silicon nitride is a promising high-index material for dense photonic circuits and applications in the visiblemidinfrared wavelength regime. Design, fabrication, and optical characterization of silicon nitride waveguides at visible-nearinfrared wavelength are presented. Finally, design and experimental results are presented for the first time for linear and focused grating couplers (GCs) at near-infrared wavelength $(900 \mathrm{~nm})$ for plasma-enhanced chemical vapor deposition silicon nitride wires $(220 \times 500 \mathrm{~nm})$ and compared with theoretical simulations. An experimental efficiency of 5.7 and $6.5 \mathrm{~dB}$ and 1-dB bandwidth of 26 and $40 \mathrm{~nm}$ are reported for the linear and focused GCs, respectively.
\end{abstract}

Index Terms-Gratings, integrated optics, optical materials, optical waveguides.

\section{INTRODUCTION}

G RATING couplers (GCs) enable efficient coupling from optical fibres to photonic integrated components (PICs) without the need of using lenses or inverted tapers [1]. They avoid expensive methods such as end-polishing and the use of lensed fibre to couple light into the waveguides, and also allows wafer-level testing of PICs. GCs with high efficiency have been successfully demonstrated for telecom wavelengths and have become a standard practice for coupling light in and out of PICs in silicon photonics [2]-[3]. GCs have also been successfully demonstrated in the telecom band in other high-index-contrast (HIC) material systems such as indium phosphide membranes [2], aluminum nitride [4], and silicon nitride $\left(\mathrm{Si}_{3} \mathrm{~N}_{4}\right)$ [5]. However, GCs for a $\mathrm{HIC}$ platform at shorter wavelengths $(400-1000 \mathrm{~nm})$ have not been investigated in detail. The visible and near-visible infrared (IR) bands are important for several applications such as sensing and spectroscopy [6], especially in a biological context given the high penetration depth of light in tissues in these bands. These shorter wavelengths also avoid water absorption band which helps in preventing cell damage at high powers. GC-based

Manuscript received June 17, 2012; revised August 4, 2012; accepted August 6, 2012. Date of publication August 10, 2012; date of current version September 12, 2012. This work was supported in part by a European Research Council InSpectra Advanced Grant.

A. Z. Subramanian, S. Selvaraja, A. Dhakal, K. Komorowska, and R. Baets are with the Photonics Research Group, Center for Nano and Biophotonics, Ghent University, Ghent 9000, Belgium (e-mail: ananth. subramanian@intec.ugent.be; shankar@intec.ugent.be; ashim.dhakal@intec. ugent.be; katarzyna.komorowska@intec.UGent.be; roel.baets@intec.ugent.be).

P. Verheyen is with IMEC, Leuven 3001, Belgium (e-mail:verheyen@ imec.be).

Color versions of one or more of the figures in this letter are available online at http://ieeexplore.ieee.org.

Digital Object Identifier 10.1109/LPT.2012.2212881

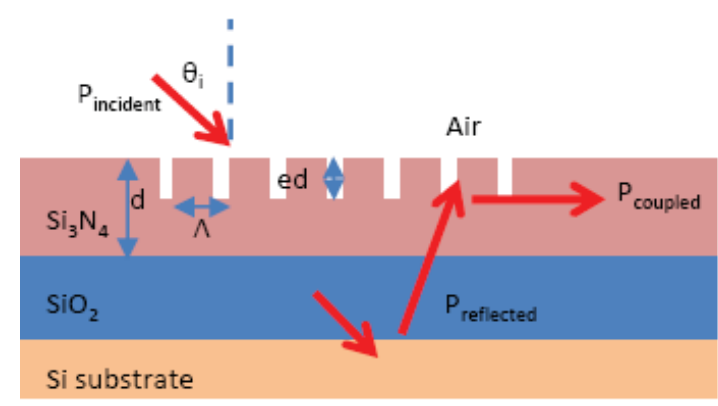

Fig. 1. Schematic diagram of the $\mathrm{Si}_{3} \mathrm{~N}_{4}$ GC. Light is incident ( $\mathrm{P}_{\text {incident }}$ ) at $\theta_{i}$ angle and coupled through GC of different etch-depth (ed) into the $\mathrm{Si}_{3} \mathrm{~N}_{4}$ waveguide $\left(\mathrm{P}_{\text {coupled }}\right)$. The period $(\Lambda)$ of the $\mathrm{GC}$ and $\mathrm{Si}_{3} \mathrm{~N}_{4}$ thickness are fixed at 630 and $220 \mathrm{~nm}$, respectively.

PICs will help in realizing efficient and easy-to-use integrated spectrometers and sensors. $\mathrm{Si}_{3} \mathrm{~N}_{4}$ is a versatile $\mathrm{HIC}$ platform for its transparency at both visible and infrared wavelengths [7]-[9]. Besides it is compatible with the well established complementary-metal-oxide-semiconductor (CMOS) process technology thereby enabling low-cost photonic devices much like in silicon photonics.

In this letter, we present the design and fabrication of singlemode $\mathrm{Si}_{3} \mathrm{~N}_{4}$ strip waveguide in $600-1000 \mathrm{~nm}$ wavelength range. Finally we report the first demonstration of the GCs at near-infrared (NIR) $(850-950 \mathrm{~nm})$ for $\mathrm{Si}_{3} \mathrm{~N}_{4}$ wires and present the coupling efficiency and its dependence on the wavelength, angle and etch depth of the grating and compare with the theoretical results.

\section{Waveguide And Grating Coupler Design}

For designing waveguides, Fimmwave (PhotonDesign) waveguide mode solver was used for simulations. A crosssection of $220 \times 500 \mathrm{~nm}$ was used to define the core of $\mathrm{Si}_{3} \mathrm{~N}_{4}$ waveguide. For this dimension, the $\mathrm{Si}_{3} \mathrm{~N}_{4}$ waveguide was singlemode in 600-1000 $\mathrm{nm}$ wavelength range. The gratings were designed for transverse electric (TE) polarization with maximum efficiency around $900 \mathrm{~nm}$, using CAMFR, an eigenmode expansion tool [10]-[11]. The GC period, fill factor (pitch/period) and number of periods was fixed at $630 \mathrm{~nm}$, 0.5 and 20, respectively. The etch depth of GCs and incident angle were left as free parameters to maximize the coupling efficiency. A schematic diagram of the GC is shown in Fig. 1.

Two types of GCs were designed-linear GC (LGC) and focused GC (FGC). The schematics of both GCs (snapshots from the mask design) are shown in Fig. 2. In case of LGC, 


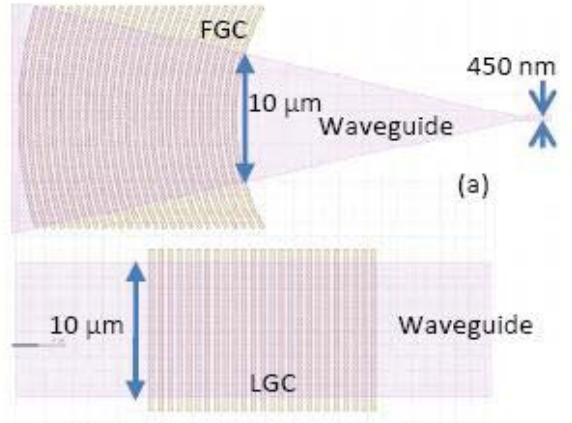

(b)

Fig. 2. Snapshots from the mask design showing (a) FGC and (b) LGC defined on top of $10-\mu \mathrm{m} \mathrm{Si}{ }_{3} \mathrm{~N}_{4}$ waveguide. The period was fixed at $630 \mathrm{~nm}$.

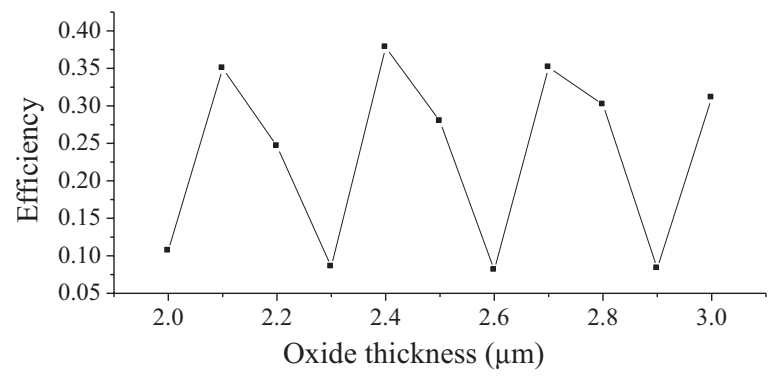

Fig. 3. Effect of buried oxide thickness on the GC efficiency as calculated by CAMFR.

two GCs at either end of the waveguide were defined on a $10 \mu \mathrm{m}$ wide waveguide which was adiabatically tapered down to a $500 \mathrm{~nm}$ wire. The total length of the waveguide was $4 \mathrm{~mm}$. A FGC focuses the light directly from a $10 \mu \mathrm{m}$ wide waveguide to a $450 \mathrm{~nm}$ wide wire. The total length of the waveguide was $6 \mathrm{~mm}$.

The underlying oxide thickness has a major influence on the coupling efficiency. Its value is chosen in such a way that the downward radiated light which gets reflected at the oxide/substrate interface $\left(\mathrm{P}_{\text {reflected }}\right.$ in Fig. 1) interferes constructively with the direct upward radiated light. This effect is depicted in Fig. 3 for the central wavelength $(900 \mathrm{~nm})$ of the coupler. Based on this result the oxide thickness was fixed at $2.4 \mu \mathrm{m}$.

\section{III. $\mathrm{Si}_{3} \mathrm{~N}_{4}$ WAVEGUIDE FABRICATION AND CHARACTERIZATION}

For the fabrication, a $200 \mathrm{~mm}$ bare $\mathrm{Si}$ wafer is used as the substrate. PECVD is used to first deposit $2.4 \mu \mathrm{m}$ of silicon dioxide $\left(\mathrm{SiO}_{2}\right)$ followed by $\sim 220 \mathrm{~nm}$ thick $\mathrm{Si}_{3} \mathrm{~N}_{4}$ deposition on top. The $\mathrm{SiH}_{4}, \mathrm{~N}_{2}$ and $\mathrm{NH}_{3}$ gas flows were optimized for $\mathrm{Si}_{3} \mathrm{~N}_{4}$ deposition at $400{ }^{\circ} \mathrm{C}$, which ensured CMOS back-end compatibility. The deposited thin film was characterised using a prism coupling method at $633 \mathrm{~nm}(\mathrm{He}-$ Ne laser) for the TE polarization. The light was coupled into the waveguide using a rutile prism into the fundamental mode of the $\mathrm{Si}_{3} \mathrm{~N}_{4}$ waveguide. The index measurements were done at different places on the sample to check the homogeneity of the deposited thin film and averaged.
After the layer deposition, the waveguide and the GCs were patterned by using $193 \mathrm{~nm}$ optical lithography and reactive ion etch process. The waveguide was deeply etched $(220 \mathrm{~nm}$ deep), and GCs were defined with different etch depths by controlling the etch duration. Photoresist was used as an etch mask for both the etch processes. After dry etching, the wafers were cleaned by using oxygen plasma and a wet chemical process. The widths of the waveguides were in the range of $500 \pm 30 \mathrm{~nm}$. Finally, some of the wafers were covered with $1.5 \mu \mathrm{m}$ of $\mathrm{SiO}_{2}$ using PECVD process. Since $\mathrm{Si}_{3} \mathrm{~N}_{4}$ does not have any absorption band near $900 \mathrm{~nm}$ wavelength band, therefore no heat treatment was applied to the $\mathrm{Si}_{3} \mathrm{~N}_{4}$ samples. At the end, the dies were cleaved from the wafer for optical characterization.

The propagation loss in the $500 \mathrm{~nm}$ wide wire was measured by cutback method using spiral waveguides with different lengths $(1,2,4$ and $7 \mathrm{~cm})$ and a bend radius of $10 \mu \mathrm{m}$. These measurements were performed for the TE polarization using a tunable laser source emitting in the range 890$910 \mathrm{~nm}$ and a singlemode fibre at this wavelength range. GC measurements were performed by coupling unpolarized light from a tungsten halogen white light source (400-1700 nm) using a singlemode fibre into the $\mathrm{Si}_{3} \mathrm{~N}_{4}$ waveguides. Another similar fibre is positioned above the output GC to collect the light into an optical spectrum analyzer. Both fibres were mounted on a goniometer-controlled stage to change the tilt angle. The coupling efficiency was determined from the fibreto-fibre transmission. The position of the fibre was optimized for the maximum transmission.

\section{RESUlts AND Discussion}

The prism coupling experiment yielded a refractive index of $2.043 \pm 0.005$ and a good uniformity with $<1 \%$ variation across the wafer. The waveguide losses in the $500 \mathrm{~nm}$ wires were measured (@900 nm) for different dies from different wafers for checking the uniformity of the fabricated devices. The unclad and clad waveguides yielded a loss of $3.5 \pm$ $1.7 \mathrm{~dB} / \mathrm{cm}$ and $2.8 \pm 0.6 \mathrm{~dB} / \mathrm{cm}$, respectively.

The GC efficiency (theoretical and experimental) as a function of incidence angle for $140 \mathrm{~nm}$ etched LGC at a wavelength of $890 \mathrm{~nm}$ is shown in Fig. 4. The maximum efficiency is achieved for an angle of $8^{\circ}$ and gradually reduces for all other angles. A fairly large $1 \mathrm{~dB}$ angle tolerance of $\sim 4^{\circ}$ is measured for this etch-depth. Similar optimisation was done for other etch-depths as well. The experimental measurement showed a similar trend as predicted by simulations but there was a difference in the absolute value as discussed later.

The LGC efficiency as a function of wavelength for different etch depths $(70,120, \& 140 \mathrm{~nm})$ is plotted in Fig. 5. The plot compares the experimental and theoretical results for the efficiencies. The efficiency plotted in Fig. 5 is calculated at optimum angle for different etch depths as shown in Fig. 4.

The efficiency of the FGC is also measured and shown in Fig. 6. The $120 \mathrm{~nm}$ etched FGC yielded the best efficiency of as high as $-5.7 \mathrm{~dB}$ and a $1 \mathrm{~dB}$ bandwidth of $\sim 23 \mathrm{~nm}$ whereas the $220 \mathrm{~nm}$ etched FGC exhibited the lowest efficiency of $-7.7 \mathrm{~dB}$ and a $1 \mathrm{~dB}$ bandwidth of $\sim 25 \mathrm{~nm}$ respectively. 


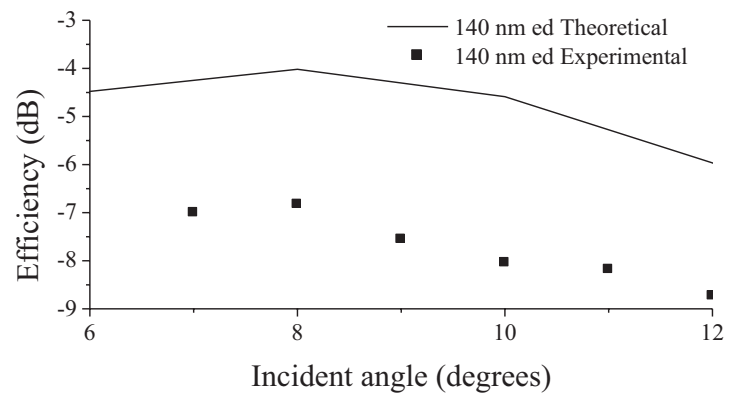

Fig. 4. LGC efficiency (theory and experiment) at $890 \mathrm{~nm}$ for $140 \mathrm{~nm}$ etched $\mathrm{GC}$ for different coupling angles.

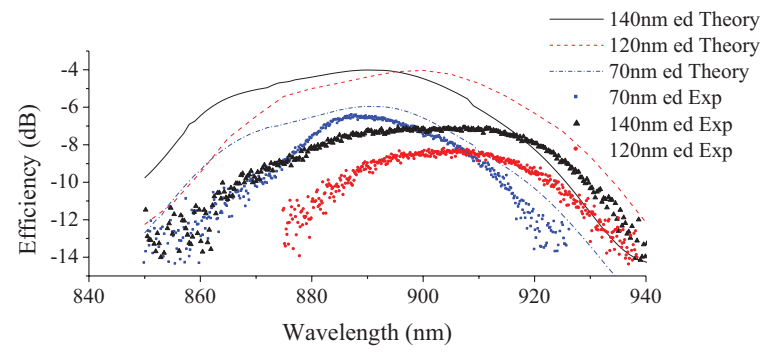

Fig. 5. LGC efficiency (theory and experiment) for different etch depths.

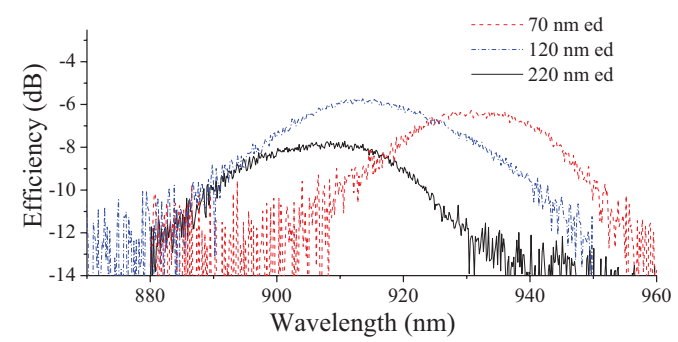

Fig. 6. FGC efficiency for 70, 120, and $220 \mathrm{~nm}$ etched GCs.

The grating strength (hence the effective grating length) and the reflection spectrum from the underlying substrate varies with the etch depth of the grating. For our experiments, the grating length was fixed at 20 periods and the difference in the reflection spectrum due to different etch depths leads to slight difference in the $1 \mathrm{~dB}$ bandwidth. Table 1 shows the efficiency and the bandwidth of FGCs with different etch depths.

The high loss in the wire is attributed to the scattering losses at the rough sidewalls as evident from the difference in the waveguide loss for clad and unclad samples. At shorter wavelengths, scattering increases and becomes more dominant and is believed to be the main source for the high waveguide loss. Therefore, further optimisation of the etch chemistry will be undertaken to smoothen the sidewall roughness and in turn reduce the losses in the waveguides.

Table 1 also compares the theoretical and experimentally determined GC efficiency and $1 \mathrm{~dB}$ bandwidth of the LGCs of different etch depths. The theoretical LGC efficiency calculations (dotted lines in Fig. 5) shows a maximum coupling efficiency of $\sim-4 \mathrm{~dB}$ for the $140 \mathrm{~nm}$ etched grating at $890 \mathrm{~nm}$ and lowest $(-5.9 \mathrm{~dB})$ for the $70 \mathrm{~nm}$ etched LGC at $890 \mathrm{~nm}$. Experimental measurements of the LGC for $70 \mathrm{~nm}$ etch depth shows reasonable agreement with the theoretical calculations.
TABLE I

EFFICIENCY AND BANDWIDTH COMPARISON FOR DIFFERENT GRATING COUPLERS AND ETCH DEPTHS

\begin{tabular}{cccccc}
\hline \hline GC & $\begin{array}{c}\text { Etch } \\
\text { depth } \\
(\mathrm{nm})\end{array}$ & $\begin{array}{c}\text { Exp } \\
\text { efficiency } \\
(\mathrm{dB})\end{array}$ & $\begin{array}{c}\text { Theoretical } \\
\text { efficiency } \\
(\mathrm{dB})\end{array}$ & $\begin{array}{c}\text { Exp 1 dB } \\
\text { bandwidth } \\
(\mathrm{nm})\end{array}$ & $\begin{array}{c}\text { Theoretical } \\
1 \mathrm{~dB} \\
\text { bandwidth } \\
(\mathrm{nm})\end{array}$ \\
\hline FGC & 70 & -7.8 & NA & $25 \pm 1$ & NA \\
FGC & 120 & -5.7 & NA & $24 \pm 1$ & NA \\
FGC & 220 & -7.7 & NA & $21 \pm 1$ & NA \\
LGC & 70 & -6.5 & -5.9 & $22 \pm 1$ & 29 \\
LGC & 120 & -8.2 & -4 & $30 \pm 1$ & 32 \\
LGC & 140 & -7 & -4 & $37 \pm 2$ & 35 \\
\hline
\end{tabular}

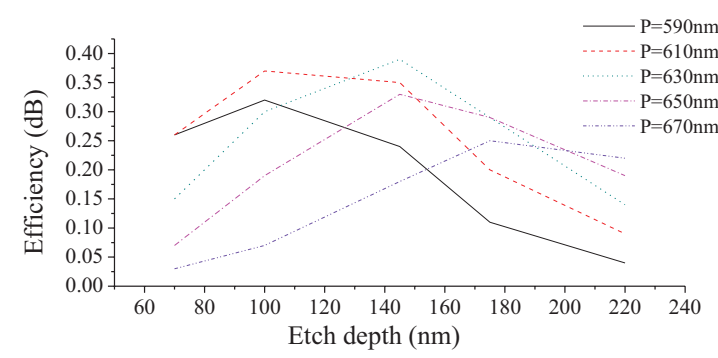

Fig. 7. Effect of period on the efficiency of GC for a given oxide thickness $(2.5 \mu \mathrm{m})$ and $8^{\circ}$ coupling angle.

The $70 \mathrm{~nm}$ etched LGC exhibited a max efficiency of $-6.5 \mathrm{~dB}$ at $888 \mathrm{~nm}$ with a $1 \mathrm{~dB}$ bandwidth of $\sim 22 \mathrm{~nm}$. The $140 \mathrm{~nm}$ etched LGC shows a slightly lower efficiency of $-7 \mathrm{~dB}$ at $907.5 \mathrm{~nm}$ with a wider $1 \mathrm{~dB}$ bandwidth of $\sim 37 \mathrm{~nm}$. The $120 \mathrm{~nm}$ etched LGC exhibited much lower efficiency $(\sim-8 \mathrm{~dB})$ and a rather poor correspondence with the theoretical predictions. There is decent correspondence between the theoretical and experimental values of $1 \mathrm{~dB}$ bandwidth for LGC, but the big difference between the experimental values for the 120 and $140 \mathrm{~nm}$ etched LGC is not fully understood yet. It is believed to be caused by some anomaly in the fabricated gratings leading to deviation in the recorded signal for this grating. Also, unfortunately the direct comparison between the $140 \mathrm{~nm}$ LGC and FGC could not be made due to damaged waveguides in the case of FGC.

The discrepancy between the experiment and theoretical results are attributed to several reasons - a) the variation in the absolute value of the buried oxide thickness $(\sim 250 \mathrm{~nm})$ leading to reduced efficiency as shown in fig. 2, b) these experiments were performed without using index matching fluid (IMF) and it is expected that using IMF would reduce the reflection losses and further improve the efficiency, c) for a given oxide thickness, a variation $( \pm 20 \mathrm{~nm})$ in the targeted grating period has an effect both on the peak wavelength and the efficiency value of the GC output as shown in Fig. 7, and finally, d) just like in waveguides, the current etch recipe is also believed to yield rough sidewalls in the GCs leading to excess scattering loss and contributes to a lower GC efficiency.

It is worthwhile to mention here that $\mathrm{Si}_{3} \mathrm{~N}_{4}$ is a deposited material and therefore it is possible to further boost the 


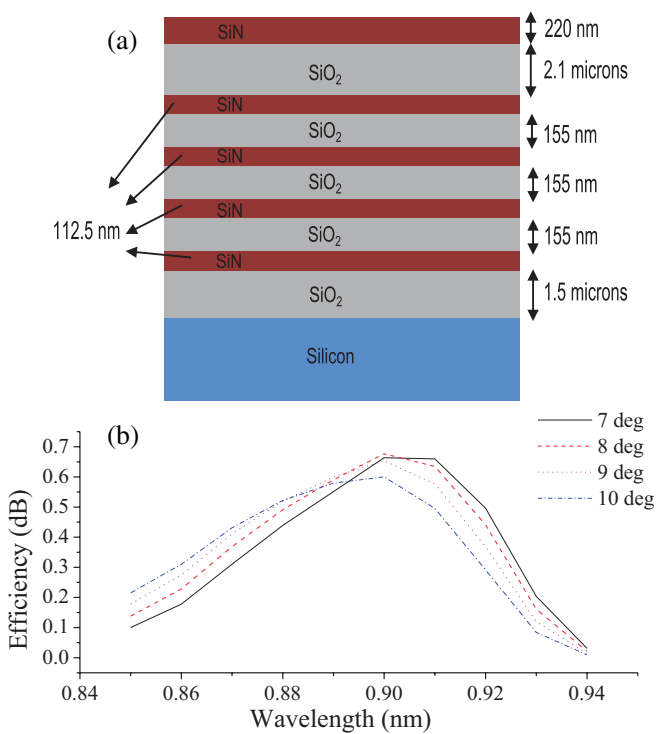

Fig. 8. (a) $\mathrm{Si}_{3} \mathrm{~N}_{4} \mathrm{GC}$ with $\mathrm{DBR}$ reflector and (b) simulated results at different coupling angles. The $\mathrm{Si}_{3} \mathrm{~N}_{4}$ and $\mathrm{SiO}_{2}$ thicknesses are fixed at 112.5 and $155 \mathrm{~nm}$, respectively. The top oxide thickness is fixed at $2.1 \mu \mathrm{m}$.

efficiency by depositing a metallic mirror [2] or distributed Bragg reflectors (DBRs) underneath the grating to boost the reflection of the downward radiated light [5], [12]. Figure 8 (top) depicts a design for a DBR reflector consisting of three alternating stacks of $\mathrm{Si}_{3} \mathrm{~N}_{4}(112.5 \mathrm{~nm})$ and $\mathrm{SiO}_{2}(115 \mathrm{~nm})$ of different thickness. On top of such a stack a $2.1 \mu \mathrm{m}$ oxide buffer layer is used to maximise the reflection of downward going wave. Fig. 8 (bottom) shows the simulated results for such a structure and the results suggest that an efficiency as high as $68 \%$ is achievable with such a design.

\section{Conclusion}

In conclusion, we have demonstrated GCs for the NIR wavelengths for the first time on a PECVD $\mathrm{Si}_{3} \mathrm{~N}_{4}$ platform using a simple tungsten halogen white light source. The LGC partially etched to $70 \mathrm{~nm}$ exhibited a best coupling efficiency of $-6.5 \mathrm{~dB}$ with a $1 \mathrm{~dB}$ bandwidth of $22 \mathrm{~nm}$, whereas the GC etched by $140 \mathrm{~nm}$ showed a much wider $1 \mathrm{~dB}$ bandwidth of $37 \mathrm{~nm}$ but a slightly lower efficiency of $-7 \mathrm{~dB}$. The FGCs were better than LGCs and efficiency as high as $-5.7 \mathrm{~dB}$ with a $1 \mathrm{~dB}$ bandwidth of $25 \mathrm{~nm}$ was achieved. The combination of $\mathrm{Si}_{3} \mathrm{~N}_{4}$ wires and grating couplers enables miniature spectroscopic devices with easy accessibility to the outside world at shorter wavelengths.

\section{ACKNOWLEDGMENT}

The authors would like to acknowledge Dr. J. Gates, University of Southampton for their prism coupling set up. They would also like to thank IMEC's p-line and J. Wouters for the fabrication of the $\mathrm{Si}_{3} \mathrm{~N}_{4}$ waveguides.

\section{REFERENCES}

[1] T. Shoji, T. Tsuchizawa, T. Watanabe, K. Yamada, and H. Morita, "Low loss mode size converter from $0.3 \mu \mathrm{m}$ square $\mathrm{Si}$ wire waveguides to singlemode fibres," Electron. Lett., vol. 38, no. 25, pp. 1669-1670, 2002.

[2] F. Van Laere, et al., "Compact and highly efficient grating couplers between optical fiber and nanophotonic waveguides," J. Lightw. Technol., vol. 25, no. 1, pp. 151-156, Jan. 2007.

[3] D. Taillaert, W. Bogaerts, P. Dumon, D. Van Thourhout, and R. Baets, "Bridging the gap between nanophotonic waveguide circuits and single mode optical fibers using diffractive grating structures," J. Nanosci. Nanotechnol., vol. 10, no. 3, pp. 1551-1562, 2010.

[4] S. Ghosh, C. R. Doerr, and G. Piazza, "Aluminum nitride grating couplers," Appl. Opt., vol. 51, no. 17, pp. 3763-3767, 2012.

[5] C. R. Doerr, L. Chen, Y. K. Chen, and L. L. Buhl, "Wide bandwidth silicon nitride grating coupler," IEEE Photon. Technol. Lett., vol. 22, no. 19, pp. 1461-1463, Oct. 1, 2010.

[6] I. M. White, J. Gohring, and X. Fan, "SERS-based detection in an optofluidic ring resonator platform," Opt. Express, vol. 15, no. 25, pp. 17433-17442, 2007.

[7] A. Gorin, A. Jaouad, E. Grondin, V. Aimez, and P. Charette, "Fabrication of silicon nitride waveguides for visible-light using PECVD: A study of the effect of plasma frequency on optical properties," Opt. Express, vol. 16, no. 18, pp. 13509-13516, 2008.

[8] J. N. Milgram, J. Wojcik, P. Mascher, and A. P. Knights, "Optically pumped Si nanocrystal emitter integrated with low loss silicon nitride waveguides," Opt. Express, vol. 15, no. 22, pp. 14679-14688, 2007.

[9] A. Gondarenko, J. S. Levy, and M. Lipson, "High confinement micronscale silicon nitride high Q ring resonator," Opt. Express, vol. 17, no. 14, pp. 11366-11370, 2009.

[10] D. Taillaert, P. Bienstman, and R. Baets, "Compact efficient broadband grating coupler for silicon-on-insulator waveguides," Opt. Lett., vol. 29, no. 23, pp. 2749-2751, 2004.

[11] P. Bienstman and R. Baets, "Optical modelling of photonic crystals and VCSELs using eigenmode expansion and perfectly matched layers," Opt. Quantum Electron., vol. 33, nos. 4-5, pp. 327-341, 2001.

[12] S. Selvaraja, et al., "Highly efficient grating coupler between optical fiber and silicon photonic circuit," in Proc. CLEO 2009, pp. 1-3, paper CTuC6. 